\title{
Mortalidad infantil por malformaciones congénitas y condición socioeconómica: el caso de la Argentina
}

\author{
Rubén A. Bronberg, ${ }^{1}$ Esperanza Gutiérrez Redomero, ${ }^{2}$ \\ María C. Alonso ${ }^{2}$ y José E. Dipierri ${ }^{3}$
}

Forma de citar Bronberg RA, Gutiérrez Redomero E, Alonso MC, Dipierri JE. Mortalidad infantil por malformaciones congénitas y condición socioeconómica: el caso de la Argentina. Rev Panam Salud Publica. 2012;31(6):469-75.

RESUMEN Objetivo. Relacionar la tasa de mortalidad infantil por malformaciones congénitas (TMIMC) y el porcentaje de muertes por malformaciones congénitas (\%MMC) con las características sociodemográficas y económicas en la Argentina.

Métodos. La población estudiada de la Argentina reside en 511 departamentos de 23 provincias, agrupadas en cinco regiones geográficas (Noroeste, Noreste, Centro, Cuyo y Patagonia). Las variables analizadas fueron la TMIMC y el \%MMC calculados a partir de los nacimientos y las defunciones del quinquenio 2002-2006. Además, se utilizaron 21 variables del Censo de Población y Vivienda del 2001 (Instituto Nacional de Estadística y Censos de Argentina) para construir el Indicador Sociodemográfico y Económico (ISDE) mediante el análisis de componentes principales. Se realizaron pruebas de comparación para valorar si aparecían diferencias significativas entre las distintas regiones y las correlaciones entre indicadores, y de estos con la latitud y longitud departamental.

Resultados. La TMIMC no presentó correlación significativa con el ISDE ni con las coordenadas geográficas. El \%MMC y el ISDE presentaron una correlación positiva significativa $(\mathrm{P}<0,05)$ en todos los niveles de organización política. El ISDE explicó $41 \%$ de la variación del $\% M M C$.

Conclusiones. La TMIMC no se asoció significativamente con la marcada heterogeneidad socioeconómica del país; los valores más elevados del \%MMC, en cambio, se observaron en las poblaciones del centro y sur del país. Dada la relación entre el \%MMC y el desarrollo socioeconómico poblacional se sugiere utilizar este indicador como una aproximación (proxy) de bienestar y calidad de vida.

Palabras clave Mortalidad infantil; anomalías congénitas; factores socioeconómicos; Argentina.

En la Argentina, en las últimas décadas se ha producido un descenso im-

\footnotetext{
1 Hospital General de Agudos “Dr. José María Ramos Mejía", Servicio de Neonatología, Área de Genética Médica y Poblacional, Buenos Aires, Argentina. La correspondencia se debe enviar a Rubén Adrián Bronberg, rabronberg@intramed.net

2 Universidad de Alcalá, Departamento de Matemáticas, Madrid, España.

3 Instituto de Biología de la Altura, San Salvador de Jujuy, Argentina.
}

portante de la mortalidad infantil y sus componentes (neonatal y posneonatal) asociado a un cambio del patrón de las causas de muerte, con un aumento de las producidas por malformaciones congénitas $(1,2)$. En el 2000, en la mayoría de los países de América Latina las malformaciones congénitas ocuparon el segundo lugar como causa de muerte en menores de un año de edad (3), y se estima que en estos países las malformaciones congénitas ocasionan entre el $2 \%$ y el $27 \%$ de la mortalidad infantil $(2,4)$. A este fenómeno, que se produce más tempranamente en los países desarrollados, se refieren Gortmaker y Wise (5) como "la alteración más profunda jamás registrada en la estructura del 
patrón de mortalidad infantil". En 1997 estos autores plantearon el concepto de "primera injusticia social" para referirse a las disparidades en la tecnología de los servicios de salud como uno de los determinantes de diferencias en la mortalidad infantil. Estos conceptos, incluso otros determinantes sociales y económicos de disparidad, también han sido analizados extensamente por diversos autores, sobre todo en los países desarrollados (6-11).

Bronberg et al. (2) analizaron el patrón de mortalidad infantil por malformaciones congénitas en la Argentina en el período 2002-2006 y concluyeron que se asemeja al observado en los países desarrollados, con disminución de la tasa de mortalidad infantil por malformaciones congénitas (TMIMC) y aumento concomitante del porcentaje de muertes por malformaciones congénitas (\%MMC). Estos autores comprobaron que la distribución espacial de la TMIMC es prácticamente uniforme, en especial a nivel regional y provincial, a diferencia del $\% \mathrm{MMC}$, que exhibe una gran variabilidad geográfica, probablemente indicativa de desigualdades socioeconómicas, educativas y culturales interprovinciales e interregionales.

Debido a que en la Argentina la información estadística de los certificados de defunción sobre las características socioeconómicas de los hogares y de los padres en menores de un año de edad es insuficiente y poco precisa, resulta difícil inferir diferencias socioeconómicas en la mortalidad infantil a partir de esta fuente de información. Alternativamente, diferenciales socioeconómicos de la mortalidad infantil pueden ser analizados relacionando las tasas $\mathrm{u}$ otros indicadores de mortalidad infantil con la información sociodemográfica y económica censal, a distintos niveles de la organización política de un país o una región (12).

El marco conceptual de este enfoque se basa en el planteo de Mosley y Chen (13), para quienes la ocurrencia de las causas inmediatas o próximas de muerte se encuentra condicionada por factores sociales, económicos y culturales a nivel macro, los cuales constituyen, a su vez, los determinantes distales de la mortalidad.

El objetivo de este trabajo fue relacionar el comportamiento espacial de la TMIMC y del \%MMC en la Argentina con las características sociodemográficas y económicas de sus poblaciones a través de la definición de un único indicador que resume estas características.

\section{MATERIALES Y MÉTODOS}

Se trata de un estudio descriptivo de tipo ecológico que recurre a dos fuentes de datos: censales y de defunción de menores de un año de edad.

\section{Cálculo de la TMIMC y del \%MMC}

Los datos proporcionados por la Dirección de Estadística e Información de Salud del Ministerio de Salud de la Argentina provinieron de los certificados de recién nacidos vivos y de defunción en menores de un año de edad correspondientes al quinquenio 2002-2006. Se utilizaron las siguientes variables: a) número absoluto de fallecidos; b) número absoluto de nacidos vivos y c) defunciones por malformaciones congénitas identificadas con los códigos Q00-Q99 de la Clasificación Internacional de Enfermedades, décima revisión (CIE-10) $(2,14)$. Con base en estos datos se calculó, independientemente del sexo, la TMIMC (número de fallecidos con malformaciones $/ 1000$ recién nacidos vivos) y el \%MMC (número de fallecidos con malformaciones/100 fallecidos) para las cinco regiones geográficas (Noroeste, Noreste, Centro, Cuyo y Patagonia), que comprenden 23 provincias y 511 departamentos de la Argentina.

Las regiones argentinas están compuestas por las siguientes provincias. Noroeste: Catamarca, Jujuy, La Rioja, Salta, Santiago del Estero, Tucumán; Noreste: Corrientes, Chaco, Formosa, Misiones; Centro: Buenos Aires, Córdoba, Entre Ríos, La Pampa, Santa Fe; Cuyo: Mendoza, San Juan, San Luis; y Patagonia: Chubut, Neuquén, Río Negro, Santa Cruz, Tierra del Fuego.

\section{Cálculo del Indicador Sociodemográfico y Económico (ISDE)}

La información concerniente a las variables sociodemográficas y económicas provino del Censo de Población y Vivienda del 2001 (Instituto Nacional de Estadística y Censos) y de la Dirección de Estadística e Información de Salud del Ministerio de Salud. Se consideraron los siguientes tipos de variables: educación, variables económicas, salud, necesidades básicas insatisfechas y componentes de la mortalidad infantil (tasas de mortalidad infantil neonatal temprana, tardía y posneonatal) (cuadro 1). Excepto los datos de mortalidad, los restantes

CUADRO 1. Variables utilizadas para la construcción del Indicador Sociodemográfico y Económico (ISDE)

\begin{tabular}{|c|c|}
\hline Variable & \\
\hline Educación & 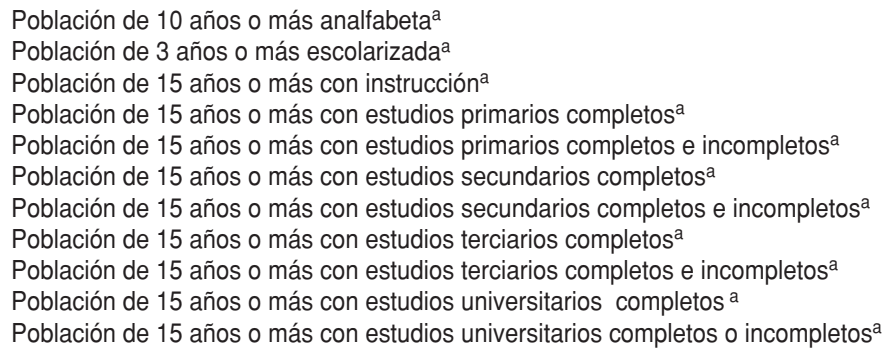 \\
\hline Económicas & $\begin{array}{l}\text { Población de } 14 \text { años o más ocupada }{ }^{a} \\
\text { Población de } 14 \text { años o más económicamente activa }{ }^{a} \\
\text { Población de jefes de hogares económicamente activa }{ }^{a} \\
\text { Población de jefes de hogares ocupados }{ }^{a}\end{array}$ \\
\hline$N B I^{b}$ & $\begin{array}{l}\text { Porcentaje de hogares con } \mathrm{NB}^{\mathrm{a}} \\
\text { Porcentaje de población con } \mathrm{NBI}^{\mathrm{a}}\end{array}$ \\
\hline Salud & Porcentaje de población sin cobertura por obra social o plan de salud o mutuala \\
\hline Mortalidad infantil & $\begin{array}{l}\text { Mortalidad infantil total ( } 0-12 \text { meses) } \\
\text { Mortalidad neonatal temprana ( } 0 \text { a } 6 \text { días) } \\
\text { Mortalidad neonatal tardía ( } 7 \text { a } 30 \text { días) } \\
\text { Mortalidad posneonatal ( } 30 \text { días a } 12 \text { meses) }\end{array}$ \\
\hline
\end{tabular}

Fuente: elaborado por los autores a partir de datos del Censo de Población y Vivienda (2001) de la Dirección de Estadística e Información de Salud del Ministerio de Salud.

a Expresado en porcentaje.

b NBI: necesidades básicas insatisfechas. 
se refieren a la población censal a nivel departamental.

A fin de determinar un único factor que resumiera el efecto de las variables socioeconómicas y demográficas se utilizó el análisis de componentes principales, con variables estandarizadas, obtenido con la siguiente combinación lineal de variables: $\mathrm{ISDE}=-0,304537 \times$ población total con analfabetismo - 0,0161336 $\times$ población total escolarizada $+0,273765 \times$ población total con instrucción - 0,299623 $\times$ población total con estudios primarios $+0,294679 \times$ población total con estudios primarios completos $+0,284667 \times$ población total con estudios secundarios $+0,274357 \times$ población total con estudios secundarios completos $+0,185107$ $\times$ población total con estudios terciarios - 0,139035 × población total con estudios terciarios completos $+0,252602 \times$ población total con estudios universitarios + $0,0338582 \times$ población total con estudios universitarios completos $+0,224578 \times$ población total económicamente activa - 0,00620258 $\times$ población total ocupada - 0,309091 × necesidades básicas insatisfechas de hogares $-0,308947 \times$ necesidades básicas insatisfechas de la población $-0,297233 \times$ población total sin obra social $-0,0542582 \times$ tasa de mortalidad infantil neonatal temprana - 0,0203826 $\times$ tasa de mortalidad infantil neonatal tardía $-0,137889 \times$ tasa de mortalidad infantil posneonatal.

El propósito de este análisis es obtener un pequeño número de combinaciones lineales de las 21 variables sociodemográficas y económicas que expliquen la mayor parte de la variabilidad de los datos; en nuestro caso tomamos la primera componente principal como ISDE, que explica $41 \%$ de la variabilidad total. El ISDE puede considerarse un indicador ampliamente representativo de las oportunidades educativas, económicas, laborales y sanitarias. Se trataría, en síntesis, de un indicador de desarrollo o de privación o carencia; a valores más altos del ISDE, mayor desarrollo y menor privación o carencia.

Para verificar la fiabilidad del ISDE como indicador de desarrollo social y económico se lo correlacionó con dos indicadores aceptados y fiables de bienestar y calidad de vida: la tasa de mortalidad infantil y el porcentaje de necesidades básicas insatisfechas (\%NBI) a nivel poblacional y en hogares. En la Argentina, estos indicadores muestran una gran heterogeneidad espacial, y sus valores más altos se observan en el norte del país $(1,15)$.

\section{Análisis estadísticos y relación de la TMIMC y el \%MMC con el ISDE}

Se calcularon los estadísticos de posición y dispersión para los indicadores TMIMC, \%MMC e ISDE en las cinco regiones geográficas (Noroeste, Noreste, Centro, Cuyo y Patagonia), conformadas por 23 provincias y 511 departamentos.

La relación entre la TMIMC y el $\%$ MMC como variables dependientes y el ISDE como variable independiente se estableció mediante análisis de correlación y regresión a nivel regional, provincial y departamental. Para detectar diferencias significativas de la TMIMC y el \% MMC entre las cinco regiones se utilizó un método no paramétrico, el contraste de Kruskal Wallis, ya que no se verificaban las hipótesis de normalidad y homocedasticidad en las cinco regiones geográficas.

\section{Análisis espacial del \% MMC, la TMIMC y el ISDE}

Para evaluar la diferenciación espacial de estos indicadores, se los correlacionó con la latitud y longitud de la localidad cabecera departamental. A fin de detectar zonas significativamente diferentes se realizó un análisis de agrupamiento a nivel departamental y provincial mediante el modelo de Poisson con el programa SaTScan v5.1 (Martin Kulldorff/ Information Management Services Inc., EE.UU.), que permite identificar agrupamientos y verificar si estos son estadísticamente significativos (16).

\section{RESULTADOS}

El ISDE presentó una correlación negativa estadísticamente significativa con la tasa de mortalidad infantil (coeficiente de correlación $\mathrm{r}=-0,310 ; P<0,01$ ), el porcentaje de población con NBI ( $\mathrm{r}=$ $-0,90 ; P<0,01)$ y el porcentaje de hogares con NBI ( $\mathrm{r}=-0,91 ; P<0,01)$; por lo tanto, constituye un indicador válido de desarrollo, o de privación o carencia.

El ISDE no se correlacionó de forma significativa con la latitud pero sí con la longitud departamental, con la cual presentó una correlación negativa estadísticamente significativa $(r=-0,488$; $P<0,01)$, observándose así la distribución preponderante de los departamen- tos más desarrollados o menos carenciados en el sur del país. Este resultado coincide con diversos antecedentes que demuestran que los fenómenos socioeconómicos (desempleo, desigualdades económicas y de bienestar, inequidad y pobreza), se explican en la Argentina por su carácter espacial heterogéneo (15).

La TMIMC no se correlacionó de forma significativa ni con la latitud ni con la longitud departamental. En cambio, el \%MMC se correlacionó tanto con la latitud $(\mathrm{r}=-0,171 ; P<0,01)$ como con la longitud ( $\mathrm{r}=-0,385 ; P<0,01$ ); estas correlaciones negativas fueron bajas, pero estadísticamente significativas. Esto indicaría que los departamentos con el mayor porcentaje de muertes por malformaciones congénitas en menores de un año de edad tenderían a localizarse hacia el este del país.

El \%MMC presentó una correlación significativa positiva con el ISDE a nivel departamental $(\mathrm{r}=0,47 ; P<0,01)$, provincial $(\mathrm{r}=0,68 ; P<0,01)$ y regional $(\mathrm{r}=0,84 ; P<0,05)$. La TMIMC no se correlacionó con el ISDE a ningún nivel de la organización política del país (departamental, regional o provincial).

En el cuadro 2 y en la figura 1 se resume la estimación de las medias y desviaciones estándares del \%MMC por región, y se observan diferencias estadísticamente significativas entre ellas $(P<$ $0,01)$ para un nivel de confianza del $95 \%$ con el contraste de Kruskal Wallis. Las regiones Noroeste y Noreste constituyen un grupo homogéneo y presentan los valores más bajos del \%MMC. En el cuadro 2 y en la figura 2 se puede observar la estimación del ISDE por regiones, y también se observan diferencias estadísticamente significativas entre ellas $(P<$ $0,01)$ con el contraste de Kruskal Wallis. En este caso, las regiones Cuyo y Patagonia constituyen un grupo homogéneo.

La comparación de las líneas de regresión del ISDE frente al \% MMC según la región presentó un $\mathrm{R}$ cuadrado $=0,45$. Dado que el valor de $P$ para las pendientes y para los puntos de corte fue inferior a 0,01, existe correlación estadísticamente significativa entre el ISDE y el \%MMC en cada una de las cinco regiones al 99\% de nivel de confianza (figura 3).

A nivel departamental se detectaron 6 agrupamientos estadísticamente significativos (cuadro 3). Los agrupamientos 1,2 y 3 , con \%MMC bajos e ISDE con valores negativos (menor desarrollo 
CUADRO 2. Media, mediana, desvío estándar y valores límites del porcentaje de muerte por malformaciones congénitas y del Indicador Sociodemográfico y Económico por región en la Argentina

\begin{tabular}{|c|c|c|c|c|c|c|c|c|c|}
\hline \multirow[b]{2}{*}{ Región } & \multirow{2}{*}{$\begin{array}{c}\text { Número de } \\
\text { departamentos }\end{array}$} & \multicolumn{4}{|c|}{ Porcentaje de muerte por malformaciones congénitas } & \multicolumn{4}{|c|}{ Indicador Sociodemográfico y Económico } \\
\hline & & Media $\pm D^{a}$ & Mediana & Mínº & Máxc & Media $\pm D E^{a}$ & Mediana & Mínb & Máx \\
\hline Centro & 216 & $25,1 \pm 12,7$ & 24,9 & 0,0 & 100,0 & $1,7 \pm 1,6$ & 1,8 & $-6,0$ & 6,7 \\
\hline Patagonia & 53 & $21,2 \pm 14,3$ & 22,2 & 0,0 & 66,7 & $0,2 \pm 3,3$ & 0,6 & $-7,6$ & 5,6 \\
\hline Noreste & 99 & $15,3 \pm 7,6$ & 16,4 & 0,0 & 28,1 & $-2,0 \pm 3,0$ & $-1,8$ & $-9,8$ & 4,2 \\
\hline Cuyo & 64 & $23,7 \pm 15,4$ & 24,1 & 0,0 & 100,0 & $0,6 \pm 2,0$ & 0,6 & $-5,3$ & 5,3 \\
\hline Noroeste & 76 & $16,6 \pm 8,7$ & 15,1 & 3,5 & 58,8 & $-2,9 \pm 2,3$ & $-2,9$ & $-10,8$ & 2,4 \\
\hline
\end{tabular}

a DE: desvío estándar.

b Mín: mínimo.

c Máx: máximo.

FIGURA 1. Variación del porcentaje de muertes por malformaciones congénitas (\%MMC) por región en la Argentina, representadas en un gráfico de diagrama de cajas

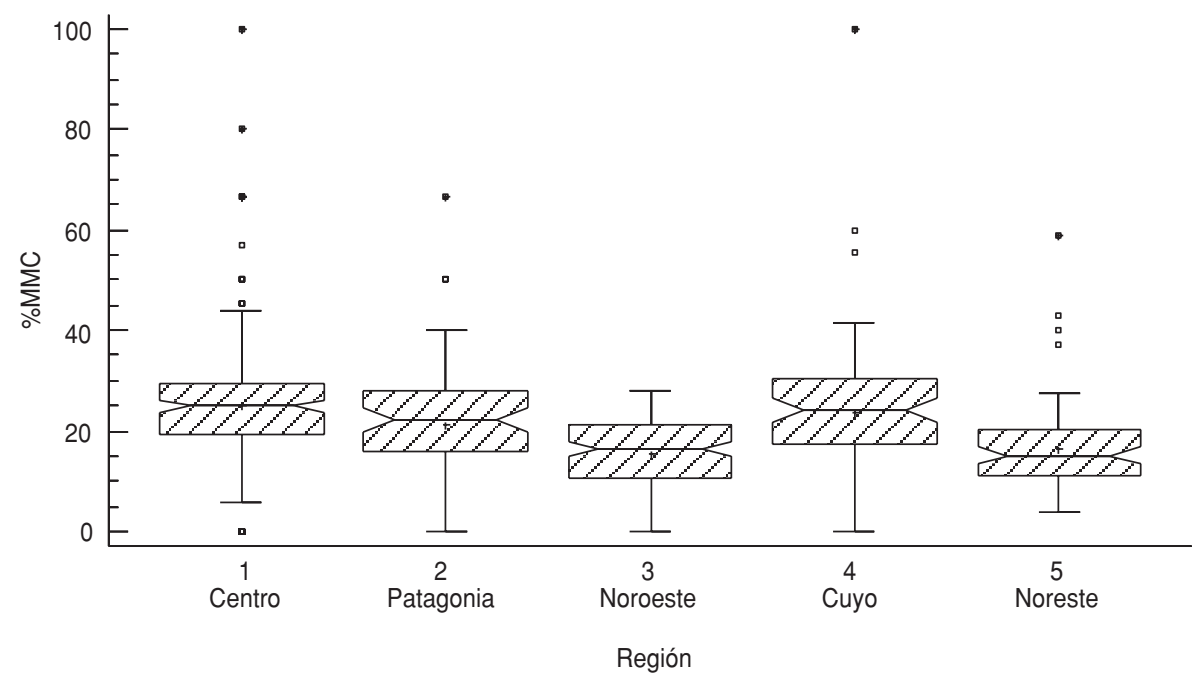

FIGURA 2. Variación del Indicador Sociodemográfico y Económico (ISDE) por región en la Argentina, representado en un gráfico de diagrama de cajas

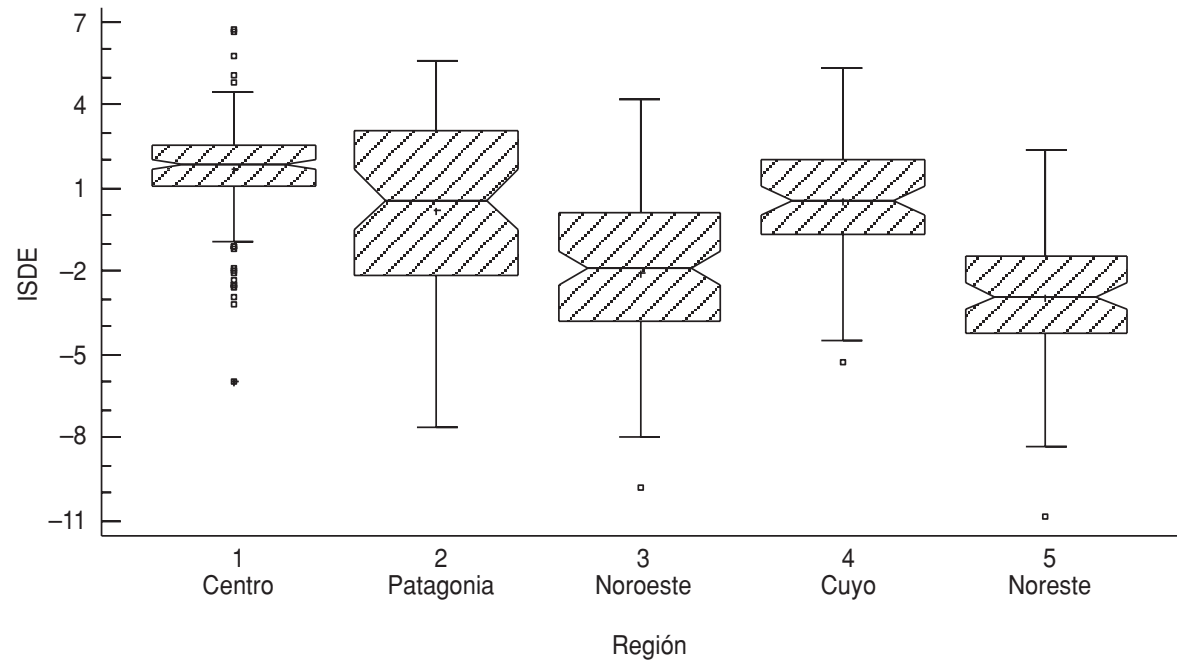

socioeconómico), se localizaron en el norte del país. Los agrupamientos 4, 5 y 6, con \%MMC altos e ISDE positivos (mayor desarrollo socioeconómico), se tivos del ISDE, fueron estadísticamente significativas con la prueba ANOVA.

\section{DISCUSIÓN}

Existen abundantes antecedentes sobre la relación inversa existente entre la condición socioeconómica de los individuos y su riesgo de muerte, tanto en los países industrializados como en aquellos en vías de desarrollo (17). En la Argentina, específicamente, el riesgo de muerte en menores de un año de edad es mayor en los grupos con mayor incidencia de NBI (18). Por este motivo, la tasa de mortalidad infantil constituye un indicador sinóptico de las condiciones sociales y de salud de las poblaciones (5).

No existen, sin embargo, muchos trabajos que analicen la relación entre el riesgo de muerte infantil por malformaciones congénitas y la condición socioeconómica de los individuos o las poblaciones. La mayoría de estos antecedentes provienen de países desarrollados (19-21) o se refieren a una malformación específica (22) y no a la mortalidad infantil por el conjunto de las malformaciones congénitas a nivel poblacional. Los indicadores de mortalidad infantil por malformaciones congénitas utilizados en este trabajo precisamente engloban a todas las malformaciones congénitas identificadas a través de la CIE-10 (Códigos Q00-Q99) (14).

En los escasos estudios que consideran a las malformaciones congénitas en su conjunto para establecer diferenciales de la mortalidad infantil según la condición socioeconómica se analiza solamente uno de los indicadores de mortalidad infantil por malformaciones congénitas utilizado en este trabajo, la TMIMC. Kurinczuk et al. (23) detectaron diferencias estadísticamente significativas en Inglaterra y Gales en la tasa de mortalidad neonatal debida a malfor- 
FIGURA 3. Regresión entre el Indicador Sociodemográfico y Económico (ISDE) y el porcentaje de muertes por malformaciones congénitas (\%MMC), según la región en la Argentina, representada en un gráfico de dispersión

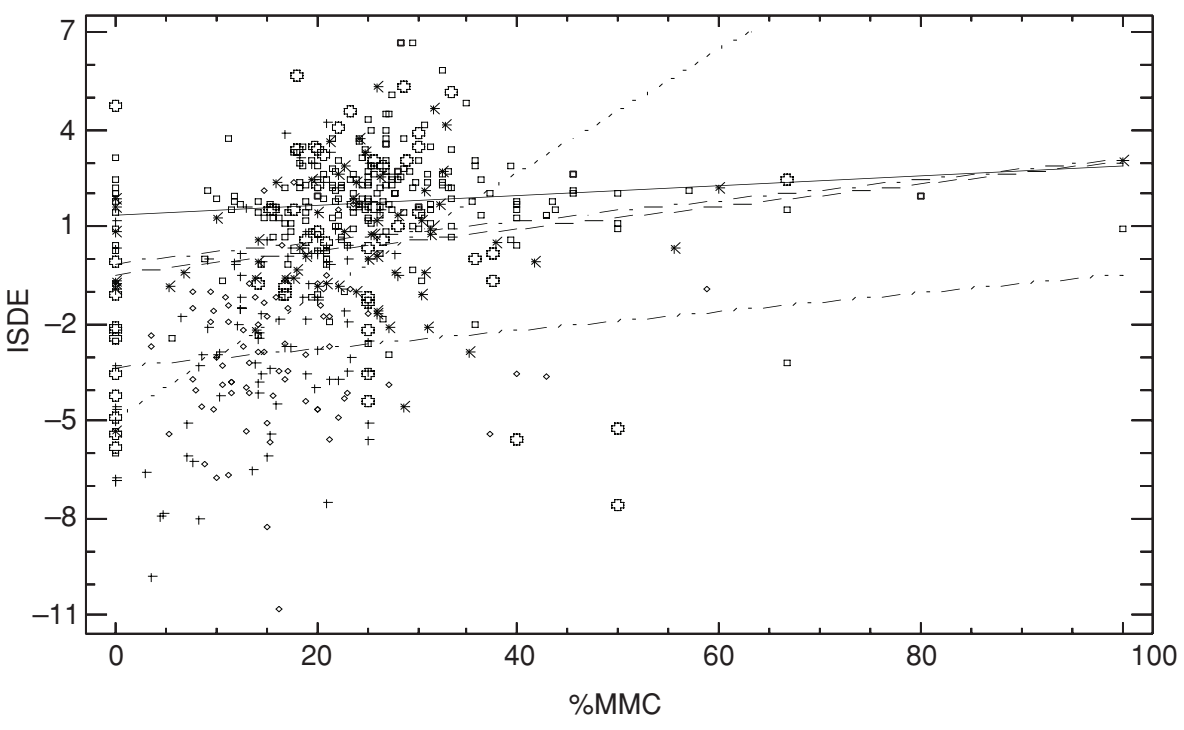

Región:

$\rightarrow$ Centro $\quad-$ - Patagonia ...-. Noroeste $-*$ - Cuyo - .. Noreste

maciones congénitas según la condición socioeconómica del recién nacido sobre la base de la ocupación de los padres. En Corea del Sur, se siguieron 1329540 niños hasta los 8 años de edad y se detectaron desigualdades en la muerte por malformaciones congénitas de acuerdo a la condición socioeconómica de los padres (24). Kurinczuk et al. (23), al igual que Parker Frisbie et al. (7) y Yang et al. (20) en los Estados Unidos, detectaron disparidades en la mortalidad infantil por malformaciones congénitas por raza, etnicidad y lugar de nacimiento materno. En estos trabajos se parte de la presunción que, en comparación con la población nativa, los inmigrantes y los grupos minoritarios presentan desventajas o se encuentran mal integrados en el sistema de salud. Sin embargo, las diferencias de mortalidad obtenidas a través de estas comparaciones deben tomarse con precaución ya que estos factores no son independientes y tienden a confundirse entre sí. Los antecedentes citados, si bien demuestran diferenciales de mortalidad infantil por malformaciones congénitas por condición socioeconómica del padre, no son comparables con este trabajo por la metodología y los resultados alcanzados.

La TMIMC en la Argentina presenta diferencias espaciales entre departamentos, provincias y regiones, pero estas no son estadísticamente significativas y tampoco se asocian significativamente a la marcada heterogeneidad socioeconómica del país. Por lo tanto, el riesgo de muerte por malformaciones congénitas de acuerdo a las características socioeconómicas del lugar donde se produce la muerte de los niños con malformaciones es semejante en cualquier punto del país. No sucede lo mismo, en cambio, con el $\% \mathrm{MMC}$, que se correlaciona positivamente con el ISDE y presenta, al igual que éste, una marcada heterogeneidad espacial con las poblaciones menos desarrolladas y con menor \% MMC situadas en el norte del país (véanse los cuadros 2 y 3). El mismo patrón se observa en todos los niveles de la organización política del país: departamentos, provincias y regiones. La distribución espacial del \%MMC constituiría una imagen en espejo de la prevalencia de causas de muerte infantil debidas a factores exógenos, particularmente aquellos detectados en el período posneonatal (enfermedades infectocontagiosas respiratorias y gastrointestinales), más frecuentes en la población en situación de pobreza o con menor desarrollo socioeconómico. Celton y Ribotta (25) analizaron el comportamiento de los componentes de la mortalidad infantil en la Argentina entre

CUADRO 3. Agrupamientos de áreas con bajo o alto porcentaje de muerte por malformaciones congénitas (\%MMC) y el correspondiente Indicador Sociodemográfico y Económico (ISDE) hallados a partir de un modelo de distribución de Poisson a nivel departamental ${ }^{\mathrm{a}}$ en la Argentina

\begin{tabular}{|c|c|c|c|c|c|c|c|c|}
\hline \multirow{2}{*}{$\begin{array}{c}\text { Área de } \\
\text { bajo o alto } \\
\% \text { MMC }\end{array}$} & \multirow[b]{2}{*}{ Agrupamiento } & \multicolumn{2}{|c|}{$\begin{array}{c}\text { Coordenadas } \\
\text { cartesianas }\end{array}$} & \multirow{2}{*}{$\begin{array}{c}\text { No. de } \\
\text { departamentos }\end{array}$} & \multirow[b]{2}{*}{ Provincias } & \multirow[b]{2}{*}{ Región } & \multirow{2}{*}{$\begin{array}{l}\% \mathrm{MMC} \\
\left(\mathrm{IC}^{\circ} \%^{\mathrm{b}}\right)\end{array}$} & \multirow{2}{*}{ 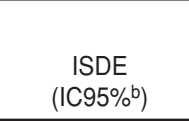 } \\
\hline & & Latitud & Longitud & & & & & \\
\hline \multirow[t]{3}{*}{ Bajo } & 1 & 578 & 1197 & 29 & Misiones, Corrientes, Formosa & Noroeste & $\begin{array}{c}12,7 \\
(11,5-14,4)\end{array}$ & $\begin{array}{c}-2,56 \\
(-3,29--1,82)\end{array}$ \\
\hline & 2 & 292 & 1263 & 42 & $\begin{array}{l}\text { Santiago del estero, Chaco, } \\
\text { Tucumán, Salta }\end{array}$ & Noreste, Noroeste & $\begin{array}{c}15,6 \\
(13,6-17,6)\end{array}$ & $\begin{array}{c}-2,98 \\
(-3,89--2,02)\end{array}$ \\
\hline & 3 & 204 & 1450 & 10 & Salta, Jujuy & Noroeste & $\begin{array}{c}10,4 \\
(4,8-16,0)\end{array}$ & $\begin{array}{c}-4,38 \\
(-6,56--2,19)\end{array}$ \\
\hline \multirow{2}{*}{ Alto } & 5 & 457 & 910 & 6 & Buenos Aires & Centro & $\begin{array}{c}28,5 \\
(26,2-29,5)\end{array}$ & $\begin{array}{c}4,29 \\
(3,17-6,66)\end{array}$ \\
\hline & 6 & 440 & 733 & 44 & Buenos Aires & Centro & $\begin{array}{c}25,4 \\
(22,8-28,1)\end{array}$ & $\begin{array}{c}2,35 \\
(2,03-2,67)\end{array}$ \\
\hline
\end{tabular}

\footnotetext{
a Estadísticamente significativos $(P<0,01)$.
}

b IC95\%: intervalo de confianza del $95 \%$. 
1950-2000 y concluyeron que las mayores desigualdades regionales se relacionaron con la reducción de la mortalidad posneonatal. Es probable que la ausencia de variación significativa de la TMIMC pueda atribuirse al comportamiento del componente neonatal de la mortalidad infantil, el cual engloba a la mayor parte de las causas endógenas, entre las que se encuentran las genéticas. De acuerdo con Celton y Ribotta, este componente se caracteriza en las últimas décadas por presentar, en relación al período posneonatal, menor variación temporal y regional. No obstante, no existen antecedentes suficientes sobre el comportamiento de los componentes de la mortalidad infantil en la TMIMC en la Argentina (2).

Sin duda el antecedente más importante, sino el único, sobre el impacto de la condición socioeconómica en las muertes infantiles por malformaciones congénitas desde una perspectiva internacional lo constituye el trabajo de Rosano et al. (4) quienes, recurriendo a los indicadores utilizados en este trabajo, TMIMC y \%MMC, analizaron los datos de mortalidad infantil por malformaciones congénitas provenientes de 36 países de Europa, Oriente Medio, América, Asia y el Pacífico Sur y los relacionaron con el producto bruto interno per cápita. Los resultados encontrados en el presente trabajo coinciden parcialmente con los de Rosano et al. (4), quienes comprobaron que la TMIMC presenta una fuerte correlación negativa con el producto bruto interno per cápita, en tanto que el \%MMC se correlaciona positivamente con este indicador de desarrollo económico.

Las malformaciones congénitas contribuyen significativamente a la morta-

1. Universidad Nacional de Lanús, Ministerio de Salud y Ambiente de la Nación, Organización Panamericana de la Salud. Atlas de Mortalidad de Argentina. [CD-ROM]. Buenos Aires: OPS/UNLa/Ministerio de Salud/ Presidencia de la Nación; 2005.

2. Bronberg R, Alfaro E, Chaves E, Dipierri J. Mortalidad infantil por malformaciones congénitas en Argentina: análisis del quinquenio 2002-2006. Arch Argent Pediatr. 2009;107(3):203-11.

3. OMS. World Health Organization World Health Report. Geneva: WHO; 1998. Pp. 43-7. Disponible en: http://www.who.int/ whr/1998/en/whr98_ch3.pdf Acceso el 12 de mayo de 2011.

4. Rosano A, D Botto L, Botting B, Mastroiacovo $P$. Infant mortality and congenital anomalies lidad infantil en las sociedades en las que las enfermedades infecciosas y las causas perinatales de muerte infantil se encuentran controladas y las deficiencias nutricionales están corregidas (26), es decir, en aquellas sociedades que han completado la transición del patrón de muertes infantiles mediante el control de las causas exógenas o evitables a través del desarrollo económico, la disminución de la pobreza y marginalidad y el mejoramiento de las condiciones sociosanitarias. La Argentina es un país aún en vías de desarrollo con profundas desigualdades y fragmentaciones socioterritoriales y esto puede visualizarse también con la distribución espacial del $\%$ MMC (véanse los cuadros 2 y 3 y la fig. 1). Dado que, de acuerdo con Rosano et al. (4), el \%MMC se relaciona positivamente con el producto bruto interno, y que en la Argentina se observa la misma relación con el desarrollo socioeconómico y la calidad de vida alcanzada por las poblaciones, este indicador de mortalidad infantil por malformaciones congénitas podría ser utilizado como un indicador sustituto (proxy) de bienestar y desarrollo socioeconómico. Esta hipótesis debería ser verificada en otros países que aún no han alcanzado o completado en todo su territorio la transición del patrón de muerte infantil hasta alcanzar el que presentan las sociedades más desarrolladas.

Entre las principales limitaciones metodológicas se debe mencionar la imposibilidad de utilizar de forma fiable la caracterización socioeconómica de los padres que presentan los certificados de defunción de menores de un año de edad, un dato de difícil interpretación

\section{REFERENCIAS}

from 1950 to 1994: an international perspective. J Epidemiol Community Health. 2000;54:660-6. 5. Gortmaker SL, Wise PH. The first injustice: socioeconomic disparities, health service technology, and infant mortality. Ann Rev Sociol. 1997;23:147-70.

6. Hummer RA. Racial differences in infant mortality in the U.S.: An examination of social and health determinants. Social Forces. 1993;72(2):529-54.

7. Parker Frisbie W, Hummer RA, Powers DA, Song SE, Pullum SG. Race/ ethnicity/nativity differentials and changes in cause-specific infants deaths in the context of declining infant mortality in the U.S.: 1989-2001. Popul Res Policy Rev. 2010;29:395-422.

8. Santos IS, Menezes MB, Mota DN, Albernaz EP, Barros AJD, Matijasevich A, Barros FC, y a menudo faltante, motivo por el cual se decidió recurrir alternativamente a la información censal. Otra limitación metodológica consistió en el desfasaje entre la información anual de los certificados de defunción y la censal, cada década, lo que podría establecer un sesgo. Con respecto a esta última información, la más completa disponible es la del censo 2001, que de todos modos no se encuentra muy alejada temporalmente de la información de los certificados de defunción que corresponde al quinquenio 2002-2006.

Se concluye que al relacionar dos indicadores de mortalidad infantil por malformaciones congénitas, la tasa (TMIMC) y el porcentaje (\%MMC), con la información sociodemográfica y económica censal a distintos niveles de las organización política de la Argentina, solamente el \%MMC mostró variaciones espaciales estadísticamente significativas en función de las características socioeconómicas de las poblaciones donde se producen las muertes por malformaciones congénitas, y fue mayor en aquellas más desarrolladas socioeconómicamente.

El principal objetivo de los gobiernos es mejorar la salud de las poblaciones, acción que se traduce en un incremento de su calidad de vida. No obstante, pese a estos esfuerzos, dentro y entre las poblaciones persisten diferencias, inequidades o disparidades en la salud de los distintos subgrupos. De acuerdo a los hallazgos alcanzados en este trabajo, al menos en la Argentina, el \%MMC constituiría un recurso estadístico sencillo y de bajo costo para obtener información y ayudar a monitorear el progreso de las diversas políticas y programas de salud.
Victora CG. Infant mortality in three population-based cohorts in Southern Brazil: trends and differentials. Cad Saude Publica. 2008;24 (Sup 3):S451-60.

9. Palma Solís MA, Álvarez Dardet Díaz C, Franco Giraldo A, Hernández Aguado I, Pérez Hoyos S. State downsizing as a determinant of infant mortality and achievement of millennium development goal. Int J Health Serv. 2009;39(2):389-403.

10. Giuffrida A. Racial and ethnic disparities in Latin America and the Caribbean: a literature review. Divers Health Care. 2010;7(2): 115-28.

11. Stephen CE. Revisiting urban health and social inequalities: the devil is in the detail and the solution is in all of us. Environ Urban. 2011;23:29-40. 
12. Preston S. The changing relation between mortality and level of economic development. Int J Epidemiol. 2007;34:484-90.

13. Mosley WH, Chen LC. An analytical framework for the study of child survival in developing countries. Pop Devel Rev. 1984;10:25-48.

14. Organización Panamericana de la Salud. Clasificación Estadística Internacional de Enfermedades y Problemas Relacionados con la Salud (décima revisión). Washington, DC: Organización Panamericana de la Salud; 1995.

15. Velásquez GA. Naturaleza y cultura en América Latina. Dinámica demográfica, calidad de vida y riesgos ambientales. Naturaleza, bienestar y malestar en la Argentina a principios del siglo XXI. Poblac Soc. 2010;17:147-73.

16. Kulldorff M, Huang L, Konty K. A scan statistic for continuous data based on the normal probability model. Int J Health Geogr. 2009;8:58.

17. Gortmaker SL. Poverty and infant mortality in the United States. Am Soc Rev. 44. 1979;(2): 280-97.

18. Buchbinder M. Mortalidad infantil y desigualdad socioeconómica en la Argentina. Tendencia temporal. Arch Argent Pediatr. 2008;106(3):212-8.
19. Petrini J, Damus K, Johnston R: An overview of infant mortality and birth defects in the United States. Teratology. 1997;56:8-10.

20. Yang $Q$, Chen $H$, Correa A, Devine $O$ Mathews T, Honein M. Racial Differences in Infant Mortality Attributable to Birth Defects in the United States, 1989-2002. Birth Defects Res A Clin Mol Teratol. 2006 (A);76:706-13.

21. Copeland G, Kirby R. Using birth defects registry data to evaluate infant and childhood mortality associated with birth defects: an alternative to traditional mortality assessment using underlying cause of death statistic. Birth Defects Res A Clin Mol Teratol. 2007;79:792-7.

22. Gerald R. Fetal and infant mortality associated with Congenital Malformation. Brit J Prev Soc Med. 1973;27:85-90.

23. Kurinczuk JJ, Hollowell J, Boyd PA, Oakley L, Brockelhurst, Gray R. Inequalities in infant mortality project briefing paper 4 . The contribution of congenital anomalies to infant mortality. Oxford: National Perinatal Epidemiology Unit, 2010. Disponible en www. npeu.ox.ac.uk/infant-mortality Acceso el 12 de mayo de 2011.
24. Kim J, Son, M, Kawachi I, Oh J. The extent and distribution of inequalities in childhood mortality by cause of death according to parental socioeconomic positions: A birth cohort study in South Korea. Soc Sci Med. 2009;69(7):1116-26.

25. Celton D, Ribotta B. Las desigualdades regionales en la mortalidad infantil de Argentina. Niveles y tendencias durante el siglo $X X$, $1^{\circ}$ Congreso de la Asociación Latinoamericana de Población, realizado en Caxambú, Brasil, 18-20 septiembre 2004. Disponible en www.alapop.org/2009/images/PDF/ ALAP2004_390.PDF Acceso el 25 de noviembre de 2011.

26. Jenkins $\mathrm{T}$. The rol of screening in the prevention in inherited disease in South Africa. S Afr Med J. 1977;51(23):832-7.

Manuscrito recibido el 31 de mayo de 2011. Aceptado para publicación, tras revisión, el 13 de febrero de 2012.
ABSTRACT

Infant mortality due to congenital malformations and socioeconomic status: the case of Argentina

Key words
Objective. Compare the infant mortality rate due to congenital malformations (IMRCM) and the percentage of deaths due to congenital malformations (\%DCM) with sociodemographic and economic characteristics in Argentina.

Methods. The Argentine study population resided in 511 departments of 23 provinces, grouped into five geographic regions (Northwest, Northeast, Central, Cuyo, and Patagonia). The analyzed variables were the IMRCM and the \%DCM calculated on the basis of births and deaths during 2002-2006 period. In addition, 21 variables were used from the 2001 Population and Housing Census (National Census and Statistics Institute of Argentina) to construct the Sociodemographic and Economic Indicator (SDEI) through the analysis of principal components. Comparison tests were carried out in order to assess the significant differences among the various regions and the correlations between indicators, and of these with the departmental latitudes and longitudes.

Results. There was no significant correlation between the IMRCM and the SDEI, nor with geographic coordinates. However, there was a significant positive correlation between the IMRCM and the SDEI $(\mathrm{P}<0.05)$ at all levels of political organization. The SDEI explained $41 \%$ of the \%DCM.

Conclusions. The IMRCM was not significantly associated with the country's marked socioeconomic heterogeneity; the highest \%DCM values, on the other hand, were observed in the populations of the central and southern areas of the country. Given the relationship between the \%DCM and socioeconomic development of the population, use of this indicator as a proxy of well-being and quality of life is suggested.

Infant mortality; congenital abnormalities; socioeconomic factors; Argentina. 